\title{
The experience of 3 years of external quality assessment of preimplantation genetic diagnosis for cystic fibrosis
}

\author{
Zandra Deans ${ }^{\star, 1}$, Francesco Fiorentino ${ }^{2}$, Anil Biricik ${ }^{2}$, Joanne Traeger-Synodinos ${ }^{3}$, Céline Moutou ${ }^{4}$, \\ Martine De Rycke ${ }^{5}$, Pamela Renwick ${ }^{6}$, Sioban SenGupta ${ }^{7}$, Veerle Goossens ${ }^{8}$ and Gary Harton ${ }^{9}$
}

Preimplantation genetic diagnosis (PGD) was first performed over 20 years ago and has become an accepted part of genetic testing and assisted reproduction worldwide. The techniques and protocols necessary to carry out genetic testing at the single-cell level can be difficult to master and have been developed independently by the laboratories worldwide offering preimplantation testing. These factors indicated the need for an external quality assessment (EQA) scheme for monogenic disease PGD. Toward this end, the European Society for Human Reproduction and Embryology came together with United Kingdom National External Quality Assessment Services for Molecular Genetics, to create a pilot EQA scheme followed by practical EQA schemes for all interested parties. Here, we detail the development of the pilot scheme as well as development and findings from the practical (clinical) schemes that have followed. Results were generally acceptable and there was marked improvement in results and laboratory scores for those labs that participated in multiple schemes. Data from the first three schemes indicate that the EQA scheme is working as planned and has helped laboratories improve their techniques and result reporting. The EQA scheme for monogenic PGD will continue to be developed to offer assessment for other monogenic disorders.

European Journal of Human Genetics (2013) 21, 800-806; doi:10.1038/ejhg.2012.244; published online 14 November 2012

Keywords: external quality assessment; preimplantation genetic diagnosis; cystic fibrosis

\section{INTRODUCTION}

Preimplantation genetic diagnosis (PGD) for severe monogenic diseases represents an important reproductive alternative to prenatal diagnosis for couples wishing to preclude the transmission of a genetic disease to their offspring. PGD was initially performed over 20 years ago, with the first children born following PGD for gender determination to exclude X-linked diseases. ${ }^{1}$ PGD avoids the need to terminate affected pregnancies, through the identification and selective transfer of unaffected in-vitro fertilisation embryos. Today it is considered an established reproductive option and is currently offered in a substantial number of specialized centres throughout the world (http://www.eshre.com).

PGD involves close collaboration between experts in both assisted reproductive techniques and genetics, and for all stages requires the highest standards in clinical and laboratory practice to preclude misdiagnosis and any adverse outcomes. ${ }^{2-4}$

With respect to monogenic diseases, PGD can theoretically be applied for any genetic disease with a definitive molecular diagnosis and/or defined linkage within a family. The quantity of sample available for the genetic analysis in PGD is minimal, and in most cases comprises no more than a single cell. Technically, single-cell diagnosis has proved to be the most difficult part of PGD. For PGD applied to monogenic diseases, methods are almost exclusively based on the PCR. Besides the limited time available between biopsy and embryo transfer (typically 24-48 h), there are inherent difficulties associated with single-cell analysis. These include total PCR failure, allelic dropout (ADO, when one of the alleles fails to amplify to detectable levels), and the likelihood of sample contamination. All technical aspects of the method should be addressed before the clinical application of any PGD PCR protocol, to ensure a reliable and accurate genotype result. ${ }^{4}$

Besides the PCR-related pitfalls, adverse outcomes subsequent to a PGD diagnosis have been attributed to tube switching during the genotype analysis, transfer of the wrong embryo(s), inappropriate protocol design and chromosomal mosaicism in the embryo(s) being analysed. ${ }^{5}$

During the two decades that PGD has been applied as a clinical diagnostic service, the PGD community has accumulated considerable experience, which indicates that the potential causes of misdiagnosis can be substantially addressed through careful assay design of optimised fluorescent and multiplexed robust PCR protocols, overall applied with the most stringent laboratory procedures to additionally

${ }^{1}$ Department of Laboratory Medicine, UK NEQAS for Molecular Genetics, UK NEQAS Edinburgh, The Royal Infirmary of Edinburgh, Edinburgh, UK; ${ }^{2}$ Genoma Laboratories, Via di Castel Giubileo, Roma, Italy; ${ }^{3}$ Laboratory of Medical Genetics, University of Athens, St Sophia's Children's Hospital, Athens, Greece; ${ }^{4}$ Service de la Biologie de la Reproduction, SIHCUS-CMCO, 19, Rue Louis Pasteur, Schiltigheim, France; ${ }^{5}$ Centre for Medical Genetics, UZ Brussel, Brussels, Belgium; ${ }^{6}$ Centre for Preimplantation Genetic Diagnosis, Guy's \& St Thomas' NHS Foundation Trust, Great Maze Pond, London, UK; 7 UCL Centre for PGD, Institute for Women's Health, University College London, London, UK; ${ }^{8}$ ESHRE Central Office, Grimbergen, Belgium; ${ }^{9}$ Reprogenetics, Livingston, NJ, USA

*Correspondence: Dr Z Deans, Department of Laboratory Medicine, UK NEQAS for Molecular Genetics, UK NEQAS Edinburgh, The Royal Infirmary of Edinburgh, Edinburgh, EH16 4SA, UK. Tel: + 44131241 6898; Fax: + 44131242 6882; E-mail: Sandi.Deans@ed.ac.uk

Received 20 April 2012; revised 14 September 2012; accepted 11 October 2012; published online 14 November 2012 
preclude contamination and tube-switching. ${ }^{4,5}$ However, the absence of universally standardsed protocols, compounded by the frequent need to set up case-specific protocols, especially for rare monogenic diseases, means that each PGD centre has its own 'in-house' protocols. This may present issues with quality control, standardisation of laboratory protocols and meeting accreditation standards ISO 15189:2007.www.iso.org/iso/home/store/catalogue_ics/ catalogue_detail_ics.htm?csnumber=42641. The ISO 15189 standards require laboratories to regularly participate in appropriate interlaboratory comparision exercises to demonstrate the quality of the service provided. It is apparent that there is a need of quality assurance within the context of PGD, preferably through some form of internationally or nationally recognised accreditation. ${ }^{6}$ Within this context, external quality assessment (EQA) is an important part of quality management and is an essential component of accreditation schemes.

EQA provides laboratories with an independent measure of the quality of their service and an evaluation against other laboratories providing equivalent services. The assessment data give service users, for example, clinics, clinicians, and patients, a mechanism to determine whether the service provided is of sufficient standard for their purposes. EQA also has an educational purpose by highlighting variation in testing protocols, differences in interpretation of results and the process of reporting results to the clinic/clinician between laboratories. Such information is crucial not only for individual laboratories to continually review and improve their service but also for professional bodies responsible for providing appropriate and up to date best practice guidelines.

United Kingdom National External Quality Assessment Services (UK NEQAS) for Molecular Genetics has been providing EQA for a range of inherited diseases since $1991 .^{7}$ It is an accredited, self-financing, non-profit making organisation and works closely with many professional bodies to ensure a high standard of laboratory testing for a wide range of molecular base tests (www.ukneqas.molgen.org.uk). Thornhill et al, ${ }^{8}$ recommended that external proficiency testing was performed annually and in the absence of a formal mechanism to deliver this assessment, UK NEQAS for Molecular Genetics and the European Society of Human Reproduction and Embryology (ESHRE) PGD consortium formed a Molecular PGD EQA Working Group with the aim to provide EQA for laboratories providing PGD for monogenic disorders.

\section{MATERIALS AND METHODS}

\section{External quality assessment}

Participation was open to all interested laboratories offering PGD for monogenic disorders. In order to provide EQA to the widest number of laboratories, an initial survey was performed, which showed that the most commonly offered autosomal recessive disorder by the interested participating laboratories was cystic fibrosis (CF) (UK NEQAS for Molecular Genetics PGD 2008 survey results). Molecular testing performed for PGD cases is not an isolated event but an integral step in the whole PGD procedure, therefore the EQA was designed to mimic the whole process as closely as possible to a 'real' PGD case. This is in line with the requirements of an EQA scheme according to ISO 15189 (ISO 15189:2007. www.iso.org/iso/home/store/catalogue_ics/ catalogue_detail_ics.htm?csnumber=42641). For each EQA run, a mock family was created comprising of a couple requesting PGD for CF and a deceased child who had been affected with CF (Figure 1).

The EQA was provided in two stages. Stage 1 involved a feasibility study using genomic DNA samples from the EQA couple and their affected child. Participants were required to perform their work up study as per their routine procedure and report whether or not they could offer PGD in their laboratory

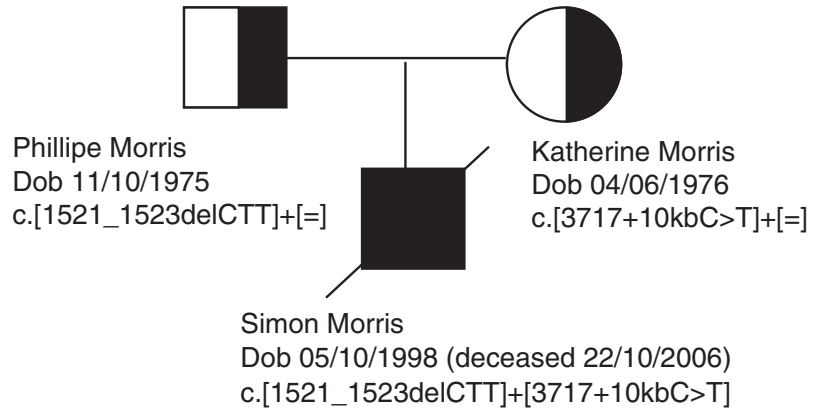

Figure 1 Example of a CF PGD EQA Case. EQA Clinical Case: Phillipe Morris and Katherine Morris requested PGD for CF. Their son, Simon Morris [deceased], was affected with CF and was a compound heterozygote for the c.1521_1523delCTT; p.Phe508del (deltaF508) mutation and the c. $3717+10 \mathrm{kbC}>\mathrm{T}(3849+10 \mathrm{kbC}>\mathrm{T})$ mutation. Phillipe was a carrier of the c.1521_1523delCTT; p.Phe508del (deltaF508) mutation and Katherine carried the c. $3717+10 \mathrm{kbC}>\mathrm{T}(3849+10 \mathrm{kbC}>\mathrm{T})$ mutation. Mutation nomenclature is given using NM 000492.3 with numbering starting at the A of the ATG initiation codon according to HGVS guidelines (www.hgvs.org). Traditional nomenclature is given in parentheses. All EQA names are fictional and any resemblance to any known individual is purely coincidental.

to the EQA couple as well as detailing their PGD strategy. Laboratories able to provide PGD went on to participate in stage 2, which required the testing of single cells to mimic the embryo cell testing performed in the laboratory. The results were submitted in the participating laboratory's usual reporting format.

All participant EQA returns were anonymised and only the UK NEQAS scheme organiser had access to the laboratory identities. All returns were marked independently against peer ratified marking criteria. The first two EQA runs were marked by the Molecular PGD EQA Working Group and the third EQA run was marked independently by three assessors with extensive experience in providing a PGD patient service. Participant submissions were assigned scores for genotyping accuracy, result interpretation and report clerical accuracy (clerical accuracy was not marked for stage 1 in runs 2 and 3 due to the use of an EQA results proforma). Each category was allocated 2.00 marks. In stage 2, each embryo was marked independently for genotyping and result interpretation and the mean score (for the five embryos) for each category was used to determine performance levels. One clerical accuracy score was given for each laboratory. Participants were given assessor feedback and a general scheme report was published following each EQA run (UK NEQAS for Molecular Genetics website publications ${ }^{9-11}$ ).

The 2008-2009 and 2009-2010 EQA schemes were provided as pilot EQAs and acceptable levels of performance were not assigned. The 2010-2011 EQA run was offered as a full scheme and therefore performance criteria produced by the Molecular PGD EQA Working Group and ratified by the UK National Quality Assurance Advisory Panel for Genetics (www.rcpath.org) were applied (http://www.ukneqas-molgen.org.uk/ukneqas/index/participants Manual/poorPerformanceCriteria.html).

\section{Selection of DNA samples for inclusion into 'mock' family undergoing PGD}

DNA samples and corresponding cell cultures were obtained from the Coriell Human Genetic Cell Repository (Camden, NJ, USA). Data from the Coriell catalogue about sex, age, race, pedigree and mutations in the CFTR gene were useful as first selection criteria. In order to create a suitable EQA family with mock embryos for each EQA run, genotype and linkage analysis was performed independently by two laboratories. Thirty-six DNA samples from six families were analysed for sets of intragenic and extragenic short tandem repeat markers closely linked to the CFTR gene. The CFTR mutations were confirmed molecularly by the Northern Genetics Service, Newcastle upon Tyne, UK. The use of samples from larger families with proband, parents and several siblings allowed the scheme organiser to set up interesting clinical cases with DNA samples from a family in stage 1 (parents carrying different CFTR 
Table 1 Overview of genotypes in CF PGD EQA cases

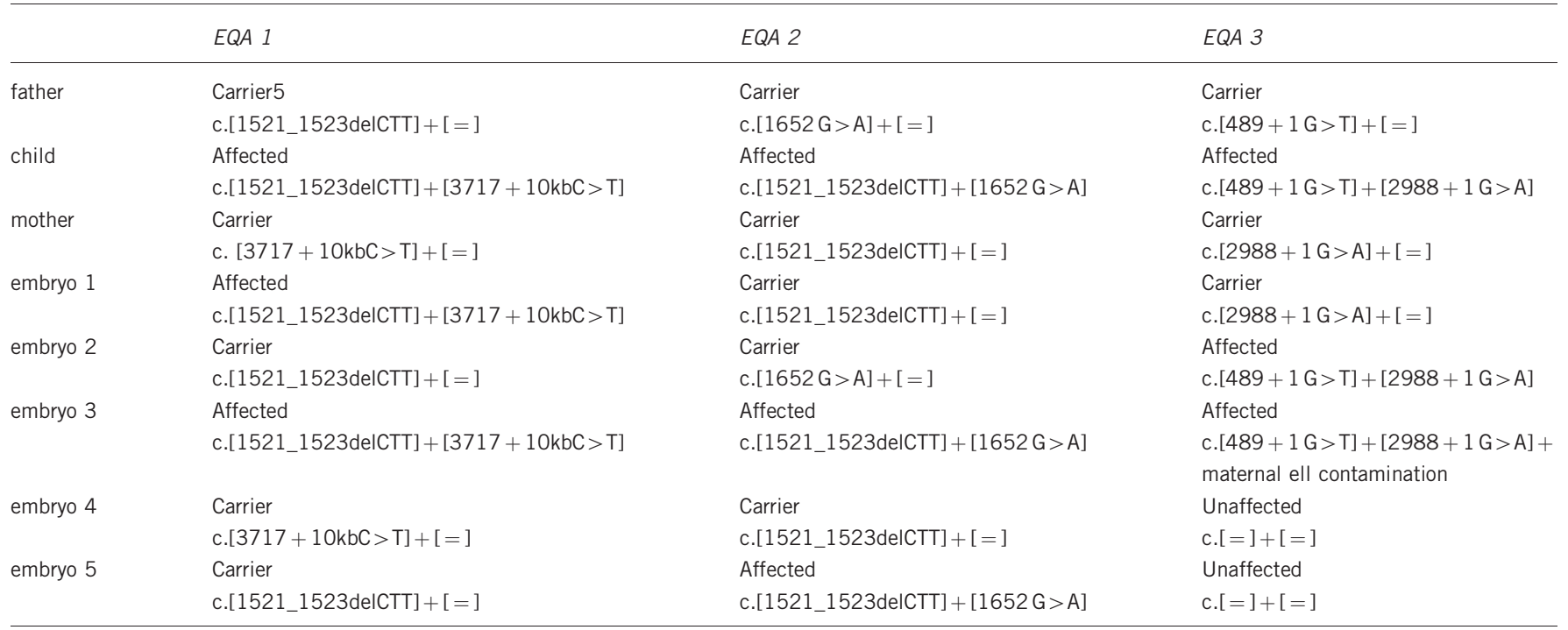

Abbreviations: $\mathrm{CF}$, cystic fibrosis; EQA, external quality assessment.

Table 2 Example of stage 1 DNA linkage analysis results

\begin{tabular}{|c|c|c|c|c|}
\hline $\begin{array}{l}\text { Chromosome location } \\
\text { According to NCBI Reference }\end{array}$ & Marker & $\begin{array}{l}\text { Father carrier of } \\
\text { c. } 489+1 G>T\end{array}$ & $\begin{array}{l}\text { Child affected c. }[489+1 \\
G>T]+[2988+1 G>A]\end{array}$ & $\begin{array}{l}\text { Mother carrier of } \\
\text { c. } 2988+1 G>A\end{array}$ \\
\hline \multicolumn{5}{|l|}{ Genome Build 36.1/hg18 } \\
\hline 111488948_111489342 & D7S523 & 227229 & 227235 & 235235 \\
\hline 115681911_115682190 & D7S486 & 142138 & 142136 & 127136 \\
\hline 116750226_116751010 & D7S23 & 180178 & 180184 & 188184 \\
\hline 116797953_116798259 & D7S633 & 201205 & 201203 & 201203 \\
\hline 116926643_116926952 & D7S677 & 111117 & 111115 & 119115 \\
\hline 116917603_116917645 & IVS1CA & 241241 & 241249 & 243249 \\
\hline 116975558_116975735 & IVS8CA & 183173 & 183175 & 173175 \\
\hline 122553259_122553350 & D7S490 & 8991 & 89103 & 97103 \\
\hline 117200939_117200998 & CFTRSTR3OAC & 276260 & 276252 & 272252 \\
\hline 117299135_117299165 & CFTRSTR15CA & 223238 & 223244 & 224244 \\
\hline
\end{tabular}

mutations and an affected child being compound heterozygous) and single lymphoblast cells in stage 2, representing embryonic cells of all possible genotypes. An overview of the genotypes of the EQA family members and mock embryos for the three EQA runs is shown in Table 1. The assessors relied on the genotype and linkage analysis results from the two validating laboratories (as shown in Table 2) during marking to assign scores for genotyping accuracy and result interpretation.

\section{Single-cell tubing/preparation and transportation}

Lymphoblast cell lines were shipped from Coriell Cell Repositories as live cultures. Once delivered, the cells were diluted with sterile $1 \times$ phosphatebuffered saline buffer pH 7.2 (Cell Signalling Technology, Danvers, MA, USA) to a suitable cell density for single-cell isolation. Lymphoblasts were then handled with a mouth-controlled fine heat-polished glass micropipette with $60-100 \mu \mathrm{m}$ diameter, prepared before biopsy with a flame source. The cells were selected and retrieved individually under visual control through an inverted phase-contrast microscope. Single lymphoblasts were first washed twice in drops of sterile phosphate-buffered saline and then transferred into sterile $0.2 \mathrm{ml}$ PCR tubes containing $1 \mu \mathrm{l}$ of phosphate-buffered saline. Blank controls were prepared from the lymphoblast's wash drops. Before proceeding to transportation, each sample was overlaid with $20 \mu \mathrm{l}$ of PCR-grade mineral oil (Sigma-Aldrich, Milan, Italy). The tubes were then placed in sterile racks and relabelled by the UK NEQAS scheme organiser to ensure subsequent blind testing by all participants. The tubes were inserted into shipping containers and distributed at environment temperature to the participating laboratories, using a single courier service.

\section{Validation of single-cell testing}

In order to validate the use of single cells in the EQA scheme, a pre-pilot assessment was performed, which involved two rounds of single-cell transportation and DNA amplification by seven laboratories experienced in single-cell amplification for PGD.

For the first round of testing, sets of 10 single cells were collected and transported to six different laboratories (one in Belgium, one in France, one in Greece, two in the UK, and one in the USA) as described above. One set of cells was not transported; rather they were split into two batches and tested independently in the Italian laboratory where the single-cell isolation had been carried out. Each laboratory selected primer sets and protocols known to work efficiently on single cells in their own laboratory. In the second round of testing, 20 isolated cells were sent to the same laboratories. These cells had been isolated at the same time as the previous sets of cells but had been stored 
at $4{ }^{\circ} \mathrm{C}$ for 6 weeks before being transported to the different laboratories for amplification.

\section{RESULTS}

Validation of single-cell testing

Overall, 80 single cells were tested in the first round of validation. A variety of techniques were applied: most laboratories froze the cells before lysis and both alkaline and enzymatic cell lysis were examined as well as multiplex PCR using a number of different Taq polymerases with and without prior whole genome amplification by multiple displacement amplification. To limit the amount of work burdened on the validation laboratories, the loci amplified were not specified and the test performed was that in use in the laboratory at that time hence a total of 42 different loci were tested and resulted in an amplification efficiency of $66.3 \%$ (53/80) (Table 3). Two out of the seven laboratories involved in this validation did not routinely accept cells from external in-vitro fertilisation centres in their PGD service. One laboratory experienced amplification problems as the box of

Table 3 Details of single-cell validation

\begin{tabular}{|c|c|c|c|c|c|c|c|}
\hline $\begin{array}{l}\text { Lab ID } \\
\text { Location }\end{array}$ & $\begin{array}{l}1 \\
\text { Italy }\end{array}$ & $\begin{array}{l}2 \\
\text { Belgium }\end{array}$ & $\begin{array}{l}3 \\
\text { France }\end{array}$ & $\begin{array}{l}4 \\
\text { Greece }\end{array}$ & $\begin{array}{l}5 \\
U K 1\end{array}$ & $\begin{array}{l}6 \\
U K 2\end{array}$ & $\begin{array}{l}7 \\
U S A\end{array}$ \\
\hline Lysis & $A L$ & $A L$ & $A L$ & PK & $A L$ & $A L$ & $A L$ \\
\hline Method & Direct multiplex PCR & $\begin{array}{l}\text { Direct } \\
\text { multiplex } \\
\text { PCR }\end{array}$ & $\begin{array}{l}\text { Direct multiplex } \\
\text { PCR }\end{array}$ & $\begin{array}{l}\text { Direct mul- } \\
\text { tiplex PCR }\end{array}$ & $\begin{array}{l}\text { Direct multiplex } \\
\text { PCR }\end{array}$ & $\begin{array}{l}\text { Round } 1 \text {, batch } 1 \\
\text { MDA then multiplex PCR } \\
\text { Round } 1 \text {, batch } 2 \\
\text { Direct singleplex PCR } \\
\text { Round } 2 \\
\text { As per round } 1 \text { batch } 1\end{array}$ & MDA then multiplex PCR \\
\hline Enzymes & $\begin{array}{l}\text { Round } 1 \text {, batch } 1 \\
\text { ABI Amplitaq } \\
\text { Round } 1 \text {, batch } 2 \\
\text { As batch } 1 \text { except cells } \\
\text { stored at } 4^{\circ} \mathrm{C} \text { for } 24 \text { hours } \\
\text { before lysis. } \\
\text { Round } 2 \\
\text { As round } 1 \text {, batch } 1\end{array}$ & $\begin{array}{l}\text { Rounds } 1 \\
\& 2 \\
\text { Qiagen } \\
\text { Multiplex } \\
\text { PCR kit }\end{array}$ & $\begin{array}{l}\text { Round 1, Batch } 1 \\
\text { Qiagen Multiplex } \\
\text { PCR kit + AL with- } \\
\text { out freezing } \\
\text { Round 1, Batch } 2 \\
\text { Qiagen Multiplex } \\
\text { PCR kit + AL with } \\
\text { freezing } \\
\text { Round } 2 \\
\text { As round 1, batch } 2\end{array}$ & $\begin{array}{l}\text { Rounds } 1 \\
\& 2 \\
\text { Qiagen } \\
\text { Multiplex } \\
\text { PCR kit }\end{array}$ & $\begin{array}{l}\text { Round } 1 \\
\text { Roche expand } \\
\text { high fidelity PCR } \\
\text { system } \\
\text { Round 2, batch } 1 \\
\text { As per round } 1 \\
\text { Round 2, batch } 2 \\
\text { Roche Expand } \\
\text { long template } \\
\text { PCR system }\end{array}$ & $\begin{array}{l}\text { Round } 1 \text {, batch } 1 \\
\text { MDA - Qiagen RepliG Midi } \\
\text { Kit then Qiagen multiplex } \\
\text { PCR kit } \\
\text { Round } 1 \text {, batch } 2 \\
\text { Roche Expand high fidelity } \\
\text { PCR system } \\
\text { Round } 2 \\
\text { As per round } 1 \text { batch } 1\end{array}$ & $\begin{array}{l}\text { Round } 1 \text {, MDA - Qiagen RepliG } \\
\text { Midi Kit then Epicentre Fail- } \\
\text { Safe enzyme } \\
\text { Round } 2 \\
\text { As per round } 1 \\
\text { Round } 2 \text {, batch } 2 \\
\text { As per round } 1 \\
\text { expect MDA for } 10 \mathrm{~h}\end{array}$ \\
\hline Loci & $\begin{array}{l}\text { D7S23 } \\
\text { D7S677 } \\
\text { CFTR IVS1 CA } \\
\text { D7S2847 } \\
\text { D7S486 } \\
\text { CFTR Exon } 10\end{array}$ & $\begin{array}{l}\text { CFTR } \\
\text { IVS8 CA } \\
\text { CFTR } \\
\text { IVS19B } \\
\text { TA } \\
\text { D7S633 } \\
\text { D7S677 } \\
\text { CFTR } \\
\text { STR30 AC } \\
\text { CFTR } \\
\text { STR15 CA } \\
\text { CFTR } \\
\text { Exon } 10\end{array}$ & $\begin{array}{l}\text { Round 1, batch } 1 \\
\text { CFTR IVS8 CA } \\
\text { CFTR IVS17B TA } \\
\text { CFTR exon } 10 \\
\text { Round 1, batch } 2 \\
\text { HTT IVS1 CA } \\
\text { D4S1614 } \\
\text { D4S3038 } \\
\text { D4S3034 } \\
\text { D4S127 } \\
\text { D4S412 } \\
\text { Round 2, batch } 1 \\
\text { As per Round } 1 \\
\text { batch } 1 \\
\text { Round 2, batch } 2 \\
\text { As per Round } 1 \\
\text { batch } 2\end{array}$ & $\begin{array}{l}\text { D11S4891 } \\
\text { D11SZ2 } \\
\text { D11S2362 }\end{array}$ & $\begin{array}{l}\text { Round } 1 \\
\text { APOC2 } \\
\text { DMPK CTG } \\
\text { repeat } \\
\text { D19S112 } \\
\text { Round 2, Batch } 1 \\
\text { As per Round } 1 \\
\text { Round 2, Batch } 2 \\
\text { Triple primed } \\
\text { PCR - DMPK } \\
\text { CTG }\end{array}$ & $\begin{array}{l}\text { Round } 1 \text {, batch } 1 \\
\text { D7S523 } \\
\text { CF_MS1 } \\
\text { D7S2554 } \\
\text { D7S2502 } \\
\text { D7S486 } \\
\text { CF_M3 } \\
\text { D7S2460 } \\
\text { IVS1CA } \\
\text { IVS8CA } \\
\text { CFSTR1 } \\
\text { D7S2847 } \\
\text { CF_MS6 } \\
\text { D7S643 } \\
\text { D7S480 } \\
\text { D7S650 } \\
\text { D7S490 } \\
\text { Round 1, Batch } 2 \\
\text { D4S127 } \\
\text { Round } 2 \\
\text { A per round } 1 \text {, batch } 1\end{array}$ & $\begin{array}{l}\text { Round } 1 \\
\text { SMN1 Exon } 7 \\
\text { SMN1 Exon } 8 \\
\text { D5S1408 } \\
\text { D5S1414 } \\
\text { HTT CAG repeat } \\
\text { HTT IVS1 CA } \\
\text { Round } 2 \\
\text { HTT CAG repeat } \\
\text { HTT IVS1 CA }\end{array}$ \\
\hline $\begin{array}{l}\text { Cells with } \\
\text { amplification }\end{array}$ & $\begin{array}{l}\text { Round } 1 \text {, batch } 1 \\
5 / 5 \\
\text { Round } 1 \text {, batch } 2 \\
5 / 5 \\
\text { Round } 2 \\
20 / 20\end{array}$ & $\begin{array}{l}\text { Round } 1 \\
8 / 10 \\
\text { Round } 2 \\
15 / 20\end{array}$ & $\begin{array}{l}\text { Round } 1 \text {, batch } 1 \\
0 / 5 \\
\text { Round } 1 \text {, batch } 2 \\
5 / 5 \\
\text { Round } 2 \text {, batch } 1 \\
10 / 10 \\
\text { Round 2, batch } 2 \\
5 / 10\end{array}$ & $\begin{array}{l}\text { Round } 1 \\
8 / 10 \\
\text { Round } 2 \\
18 / 20\end{array}$ & $\begin{array}{l}\text { Round } 1 \\
4 / 10 \\
\text { Round 2, batch } 1 \\
9 / 10 \\
\text { Round 2, batch } 2 \\
4 / 10\end{array}$ & $\begin{array}{l}\text { Round } 1 \text {, batch } 1 \\
0 / 10 \\
\text { Round } 1 \text {, batch } 2 \\
8 / 10 \\
\text { Round } 2 \\
18 / 20\end{array}$ & $\begin{array}{l}\text { Round } 1 \\
10 / 10 \\
\text { Round 2, batch } 1 \\
10 / 10 \\
\text { Round 2, batch } 2 \\
8 / 10\end{array}$ \\
\hline
\end{tabular}


transported cells was accepted with other post and unpacked centrally as for other samples sent to the centre for routine genetic analysis. This showed the importance of including clear instructions for the initial processing of the cells before amplification.

In the second round of testing, 140 cells were analysed (Table 3). Amplification was carried out at 35 loci, again using multiple brands of Taq polymerase resulting in amplification efficiency of $70.7 \%$ (99/140). As all the laboratories managed to achieve amplification in over two third of the cells using a variety of methodologies and a wide range of loci, the transport of single cells for the EQA was considered feasible. However, as the efficiency of amplification varied between groups and within groups in the two rounds of testing, it was decided that EQA participating laboratories should not be penalized for a lack of amplification. The single-cell testing in the EQA would therefore mainly examine the centre's ability to make a robust diagnosis based on the amplification results achieved. Where information is limited due to poor amplification an 'unable to state a diagnosis' is the most acceptable result.

\section{Results of three rounds of PGD EQA}

In the scheme's first year, 12 laboratories completed feasibility studies to determine if PGD could be offered to the couple (Table 4). Eleven out of the twelve participating laboratories were able to offer PGD. One laboratory did not have an optimised protocol for the informative markers for single-cell analysis and therefore, could not offer PGD and did not take part in stage 2. All eleven laboratories participating in stage 2 used a linkage strategy; nine laboratories included direct detection of the paternal mutation by fragment analysis and two laboratories also used real-time PCR or minisequencing to detect the maternal mutation. Six laboratories encountered genotype errors, mainly from laboratories using marker IVS17bta, which has a very broad allele range; this resulted in failed calling of large alleles and wrongly assumed homozygosity. This was considered a non-critical error as the resultant conclusion was that the marker was uninformative and of no further use in linkage analysis. Laboratories who failed to identify the larger alleles were given feedback about the informativity of this marker and no marks were deducted.

For the single-cell testing, if partial genotyping results were reported or no results were obtained, then full interpretation marks were assigned as long as the interpretation was correct according to the genotyping results reported. There were no errors in determining the genotype of all five embryos and no interpretation errors were made regarding the suitability of the embryo for transfer. Six out of the 11 laboratories obtained genotyping results for all five embryos and a seventh laboratory obtained results for four embryos. Two laboratories could not produce any results and contacted the scheme organiser before the report submission deadline. These two laboratories were provided with embryo haplotype data and asked to interpret the results and report in their normal manner. No genotyping score was assigned to these participants. Two laboratories obtained results for two out of the five embryos. These participants were asked to report the results they had obtained and were not penalized for failing to obtain results for the other three embryos. Both laboratories reported that the embryos for which no results were available should not be transferred and therefore were given full interpretation marks. Both of these laboratories suggested that the conditions in which the single cells were provided were not optimal for their laboratory testing.

All participants lost some points for interpretation in both stages of the EQA with the majority of marks lost for the omission of error rates, the use of non Human Genome Variation Society (HGVS) nomenclature (http://www.hgvs.org/mutnomen/) and no reference sequence being stated. Four laboratories lost marks for clerical accuracy of submitted reports, mainly for not providing two unique identifiers for each patient. Name and date of birth were used by the majority of laboratories as identifiers; however, marks were not deducted if other unique identifiers were used. Laboratories also lost marks for not stating which samples were tested, not indicating a name or signature of the report authoriser or giving the report an issue date. Professional best practice guidelines recommend these are included in a molecular report. ${ }^{12,13}$

In the scheme's second year, 10 laboratories rejoined to scheme along with five new participants (Table 4). Genotyping was generally performed to a high standard for stage 1 with only four laboratories losing marks for haplotyping errors. Four laboratories lost points in the stage 1 interpretation category; the majority of marks lost were due to not stating the limitations of the test. In this and the subsequent year, a results proforma was supplied for entering results to streamline marking. This eliminated the ability to assess clerical accuracy of feasibility reports but any clerical inaccuracies within the proforma were fedback to the laboratory. Also the laboratory reports would be fully assessed for stage 2 .

All 15 participating laboratories reported that they were able to offer PGD to the EQA couple and completed the single-cell testing. There were no errors in determining the genotypes and no interpretation errors regarding the transfer status of the embryos (Table 4). Twelve out of 15 laboratories were able to submit results for all five embryos. One laboratory did not obtain a result for one embryo but did report results for the other four embryos. One did not obtain a result for three embryos but did report results for the other two embryos and one did not obtain a result for any embryo.

In the third year, 12 of the previous year's participants rejoined the scheme and 3 new laboratories signed up to participate (Table 4). All reported that they were able to offer PGD to the EQA couple and genotyping was performed to the highest standard yet. One laboratory submitted incorrect haplotype analysis results for the parental samples for stage 1 as a result of an error when completing the EQA

Table 4 Mean scores obtained for three EQA rounds

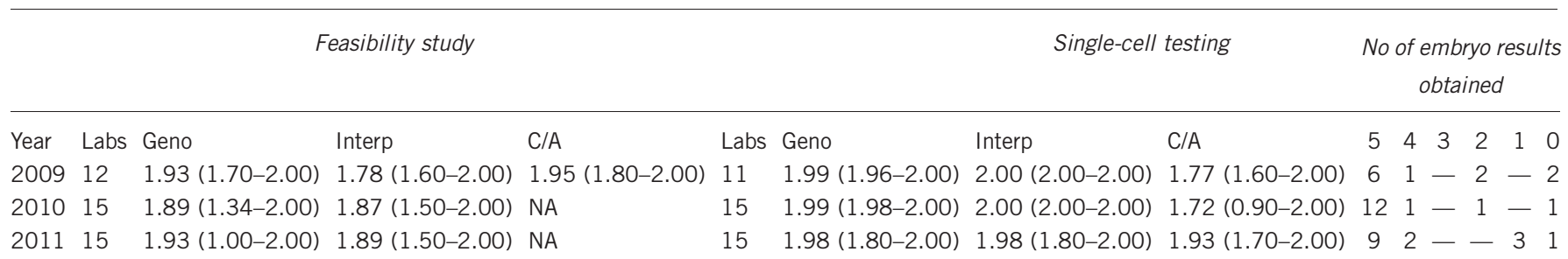

For each year the number of participating laboratories is given, along with the mean marking scores for each section and finally the number of embryos for which laboratories obtained results. The range of scores are given in brackets. Geno, mean genotyping score, Interp, mean interpretation score; C/A, mean clerical accuracy score; NA, not applicable. 
results proforma. Three laboratories lost marks for interpretation and as observed in previous years, the majority of marks lost in the interpretation category were due to not stating the limitations of the test. Some laboratories discussed limitations but $33 \%$ of participants did not state a figure to determine the test limitations. Figures quoted ranged from $<1 \%$ to $<10 \%$ with the majority stating $<1 \%$. The actual error rate given was not marked; the assessors were looking for indication that PGD results cannot be $100 \%$ sure.

Assessors also felt that the correct marker names should be used. No marks were deducted if incorrect marker names were used but a comment was made on the individual laboratory score report. It is important to give some information as to the physical and genetic location of markers (especially if locally designed markers are used) and a reference genome build is required. Compared with the reports submitted in previous years, there was an increase in the number of laboratories using HGVS mutation nomenclature (http:// www.hgvs.org/mutnomen/) and the use of a correct reference sequence.

In this third year, maternal contamination was introduced in embryo 3, by putting two cells (affected embryo and maternal) in a tube and the corresponding blank tube contained a maternal cell. Five laboratories reported this embryo as affected and did not mention maternal cell contamination. After much deliberation, the assessors felt that as they could not be certain both cells were present in the tube being tested, then these laboratories could not be marked down for the genotyping for this embryo. As the correct interpretation of the affected result was 'no transfer recommended', participants stating this were given full interpretation marks whether they mentioned the maternal contamination or not. As partial results are classed as 'did not obtain a result', the embryo 3 testing resulted in many laboratories being deemed as 'did not obtain a result'. Nine out of 15 laboratories submitted genotyping results for all five embryos. Two laboratories did not obtain a result for one embryo but did report results for the other four embryos. Three laboratories did not obtain a result for four embryos but did report results for the other embryo and one laboratory did not obtain a result for any embryo (Table 4). As seen in the previous EQA schemes, the majority of marks were lost for the omission of error rates and no reference sequence being stated. This was mainly observed in laboratories who had not previously participated in a PGD EQA scheme.

\section{DISCUSSION}

As the development of accreditation schemes in the field of analytical laboratories, EQA has become an integral part of quality management. Many national and/or international EQA schemes are available for laboratories performing molecular genetic analysis of inherited disease but only apply to genetic testing of constitutional or prenatal DNA samples. The working group for PGD EQA for monogenic diseases set up the first EQA scheme for PGD using single cells to resemble as closely as possible the clinical material routinely tested in the participating laboratories. The scheme aimed to test a laboratory's ability to carry out the whole PGD process: feasibility work-up for PGD, technical ability to do single-cell PCR, interpretation and reporting of results.

Before sending samples to participating laboratories, the EQA working group validated the samples and tested the efficacy of PCR on transported single cells. While amplification efficiency was not consistent, all laboratories had successful amplification in at least half of the cells they received using a variety of methodologies.

The first pilot scheme confirmed that the EQA scheme designed was coherent and appropriate and that the use of single lymphoblast cells in proficiency testing shows great potential for future EQA schemes. Participating laboratories in all EQA rounds have performed genotyping to a high standard and there were no errors in specifying whether or not PGD was possible. There were no errors in stage 2 single-cell testing in assignment of embryo status in the final reports when amplification was possible. Laboratories who obtained no results on 'mock embryos' were not penalised in genotyping as we could not be sure that the condition of the single cells after transportation was appropriate for testing or whether the conditions in which the cells were supplied were compatible with their local use. Of course, a laboratory that fails to amplify the supplied single cells could have a problem in the laboratory independent from shipping conditions that led to the failure. Issues of this sort are important to realise and report within an EQA scheme and are being monitored in future offerings of this EQA. This is the reason why these laboratories could request to be provided with embryo genotype/haplotype data to interpret the results and report in their normal manner; there were no errors in assignment of embryo status. These results reflect well on the ability of the participating laboratories to perform PGD, and that single cells for EQA can be successfully distributed.

These EQA schemes identified an enormous variation in the content of feasibility study reports and PGD case reports, when they existed. For both stages, the majority of marks were lost for the omission of error rates, the use of non HGVS nomenclature, the lack or reference sequence and clerical errors. Much discussion was used by the Scheme Assessors to provide fair and workable marking criteria.

Following a review of the first year of the pilot EQA, the Molecular PGD EQA Working Group decided that it was relevant to assess the PGD strategy that would be used by each laboratory. However, when assessment of strategies was initiated, it became apparent that it would be difficult to assess such strategies without having coherent guidelines to follow. An EQA scheme should not expect participants to alter their routine testing for EQA material and therefore participants in this pilot were not expected to invest time and effort in working up a PGD strategy for the EQA couple. Although laboratories had answered that they could offer PGD to the couple, some were offering strategies that would not be offered to a real clinical referral so as to participate in the scheme. It was acknowledged that PGD laboratories do spend time to maximise their PGD assays in order to provide the best possible test for their patients. Therefore, it was decided that the testing strategy would not be marked with respect to whether or not PGD could be offered based on the informativity of the markers used. Laboratories were offered the chance to participate in and be scored for round 1 of the EQA without following through on testing single cells if they would not ordinarily offer this testing to their patients or clinical referring laboratories.

Although there was an improvement in the number of laboratories able to obtain results on single cells on stage 2 throughout the EQA runs, one participant continually failed to amplify the unknown samples supplied by the EQA scheme whereas control samples were properly amplified. This shows that tubing procedure used in the EQA scheme is adapted for most, but not all PGD laboratories. Shipping and handling of samples between the EQA provider and the participating laboratories is probably the most difficult aspect to control and monitor. However, the Molecular PGD EQA Working Group decided that it was important to maintain this type of scheme with actual analysis of samples (wet EQA) as compared with a theoretical-based test.

There was also an observed improvement in the content of the reports over the 3-year period indicating that scheme 
recommendations were followed by participating laboratories. The majority of marks lost in the interpretation category were due to not stating the limitations of the test. The aim of including the limitations of the test is to give an indication of the accuracy and efficiency of the protocol that will be applied. A major limitation of all protocols is the genetic distance of informative markers flanking the mutation(s), and ideally laboratories could state the relative position and genetic distance/ physical distance of markers from the gene. In-house singlecell testing of PCR amplification and ADO rates for each locus in the protocol could also be included in workup reports. This gives an indication of the overall efficiency of the protocol and the accuracy of direct mutation testing results. Thus, depending upon the strategy used an estimate of the risk of a misdiagnosis based upon the chance of recombination and ADO could be made.

For stage 2 of the 2010-2011 EQA an additional difficulty was introduced, that is, a maternal contamination in one embryo (and a contaminated corresponding blank sample). Five out of fifteen laboratories did not mention maternal cell contamination but gave a correct interpretation that this embryo should not be transferred. It is surprising that these laboratories failed to detect maternal contamination as they genotyped the remaining embryos to a high standard. Furthermore, the contamination of the blank provided should have indicated to the laboratory the possibility of the presence of maternal cell contamination. It was not clear if all of these laboratories had not tested the blank supplied. This case highlights the importance of testing blanks in this case scenario. Several explanations for failing to report the contamination could be: inability to detect an existing contamination, non reporting or real absence of contamination that could have occurred if only one affected cell was tubed. As the scheme could not be certain that two cells (embryo and maternal) were present in the tube being tested, it was decided that we could not mark these laboratories for genotyping for this embryo. If the genotypes from the affected cell (embryo) and the maternal cell were detected, then from the linkage analysis results, it was not possible to determine whether the embryo was actually affected or a carrier of the paternal CF mutation.

This third EQA scheme also pointed out that genotyping marking is a difficult issue. Actually, even if embryo status was determined properly, genotypes were often reduced due to ADO for some loci. This is particularly true when a high number of loci were tested, either after whole genome amplification or by multiplex PCR with numerous loci. It is well known that ADO rate is increased with the number of loci amplified. So even if interpretation is correct and embryo status well established, the genotype will not be complete. The scoring of the genotype is not adapted to these highly efficient methods, but to more simple protocols and will be modified for future schemes.

Overall participation in the molecular PGD EQA scheme has resulted in laboratories reviewing and improving the content of their reports both for the feasibility work up study performed before a PGD case is undertaken and also the report issued for the PGD case itself. The EQA scheme has provided laboratories with assurance that the service they offer is comparable to other service providers and they are obtaining high EQA scores for haplotype analysis and subsequent interpretation of the results in the context of a PGD case. The future EQA schemes will be proposed for other monogenic disorders to allow participation of new laboratories. According to the top ten list of monogenic PGD indications in ESHRE PGD consortium, it was decided that the next EQA scheme will be based on a fragile $\mathrm{X}$ syndrome case. Other disorders will follow.

\section{CONFLICT OF INTEREST}

The authors declare no conflict of interest.

\section{ACKNOWLEDGEMENTS}

We would like to thank the members of all the participating laboratories and acknowledge the funding from the UK Clinical Pathology Accreditation Trust for the initial pilot EQA. Thank you also to Coriell Cell Repositories, USA for their permission to use the samples for the purposes of this EQA scheme, along with the staff of the Northern Genetics Service, Newcastle upon Tyne, UK and Genoma, Italy, for their help and support of the EQA scheme.

1 Handyside AH, Kontogianni EH, Hardy K, Winston RM: Pregnancies from biopsied human preimplantation embryos sexed by Y-specific DNA amplification. Nature 1990; 344: 768-770.

2 Harton G, Braude P, Lashwood A et al: ESHRE PGD consortium best practice guidelines for organization of a PGD centre for PGD/preimplantation genetic screening. Hum Reprod 2011; 26: 14-24.

3 Harton GL, Magli MC, Lundin K, Montag M, Lemmen J, Harper JC: ESHRE PGD Consortium/Embryology Special Interest Group-best practice guidelines for polar body and embryo biopsy for preimplantation genetic diagnosis/screening (PGD/PGS). Hum Reprod 2011; 26: 41-46.

4 Harton GL, De Rycke M, Fiorentino F et al: ESHRE PGD consortium best practice guidelines for amplification-based PGD. Hum Reprod 2011; 26: 33-40.

5 Wilton L, Thornhill A, Traeger-Synodinos J, Sermon KD, Harper JC: The causes of misdiagnosis and adverse outcomes in PGD. Hum Reprod 2009; 24: 1221-1228.

6 Harper JC, Sengupta S, Vesela K et al: Accreditation of the PGD laboratory. Hum Reprod 2010; 25: 1051-1065.

7 Ramsden SC, Deans Z, Robinson DO et al: Monitoring standards for molecular genetic testing in the United Kingdom, The Netherlands and Ireland. Genet Test 2006; 10: $147-156$.

8 Thornhill AR, de Die-Smulders CE, Geraedts JP et al: Best Practice guidelines for clinical preimplantation genetics diagnosis (PGD) and preimplantation genetic screening (PGS). Hum Reprod 2005; 20: 35-48.

9 Deans Z: PGD pilot EQA scheme report. UK NEQAS for Molecular Genetics website publications 2009; www.ukneqas-molgen.org.uk.

10 Deans Z: PGD pilot EQA scheme report. UK NEQAS for Molecular Genetics website publications 2010; www.ukneqas-molgen.org.uk.

11 Deans Z: PGD EQA scheme report. UK NEQAS for Molecular Genetics website publications 2011; www.ukneqas-molgen.org.uk.

12 Treacy RJL, Robinson DO: Best Practice Guidelines for reporting molecular genetics results 2011; www.cmgs.org.uk.

13 Swiss Society of Medical Genetics best practice guidelines on reporting in molecular genetic diagnostic laboratories in Switzerland 2003; www.ssgm.ch. 